\title{
Primary laparoscopic pull-through procedure for Hirschprung disease at a low resource setting: Experience and early results
}

\author{
Chandima P Suriyarachchi ${ }^{1}$, H M I.Sampath Bandara Herath ${ }^{2}$, Ashan C A Pathirana ${ }^{3}$, Mirna Manual $^{2}$
}

Sri Lanka Journal of Child Health, 2012; 41(2): 76-78

\begin{abstract}
Objective: To evaluate the feasibility of performing the single stage primary laparoscopic pullthrough (PLPP) surgery for Hirschprung disease (HD) to avoid the morbidity associated with traditional staged multiple surgery.
\end{abstract}

Method: PLPP was performed on 12 patients with histologically diagnosed, uncomplicated HD below the age of 4 years since April 2010. Adequate bowel preparation was achieved prior to surgery. Four-port video laparoscopy was performed. The transition zone was identified with biopsies and confirmed with frozen section. Colon and rectum were mobilized close to its wall up to the pelvic floor. Rectal prolapse was induced intussuceptically and the rectum transected $2 \mathrm{~cm}$ above the dentate line. Colon was pulled down through anus and divided at biopsy site level. Colo-anal anastomosis was performed over a rectal tube.

Results: A noteworthy improvement of bowel habits and general well being was expressed by parents during follow-up assessment. Early results from our study, as well as the long-midterm results from elsewhere in the world, suggest that the PLPP is superior to the traditional staged procedure.

Conclusion: PLPP is a better, safer and more effective approach of treating HD.

(Key words: Hirschprung disease, primary laparoscopic pullthrough)

\section{Introduction}

The treatment of Hirschprung disease (HD) has always been a challenging task for the paediatric surgeon and the recent trend is towards early surgery with fewer stages. A single stage primary pull through surgery avoids massive disfiguring scars

${ }^{1}$ Consultant Paediatric Surgeon, ${ }^{2}$ Senior Registrar Paediatric Surgery, ${ }^{3}$ Medical Officer Paediatric Surgery, Lady Ridgeway Hospital for Children, Colombo, Sri Lanka

(Received on 16 August 2011: Accepted after revision on 29 September 2011) over the abdomen (Figures 1 and 2), repeated hospital admissions with associated social and psychological problems and multiple surgical procedures under anaesthesia. Most importantly this procedure avoids a requirement for a colostomy with its associated morbidity. This is more relevant to Sri Lanka as paediatric surgical services are confined to very few centres as postoperative care of colostomy is a daunting task.

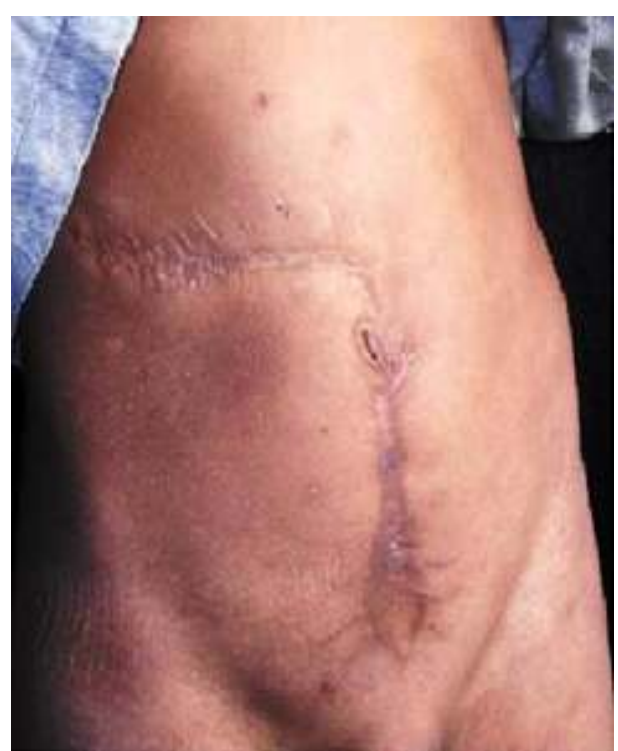

Figure 1: Outcome of traditional staged surgery for HD

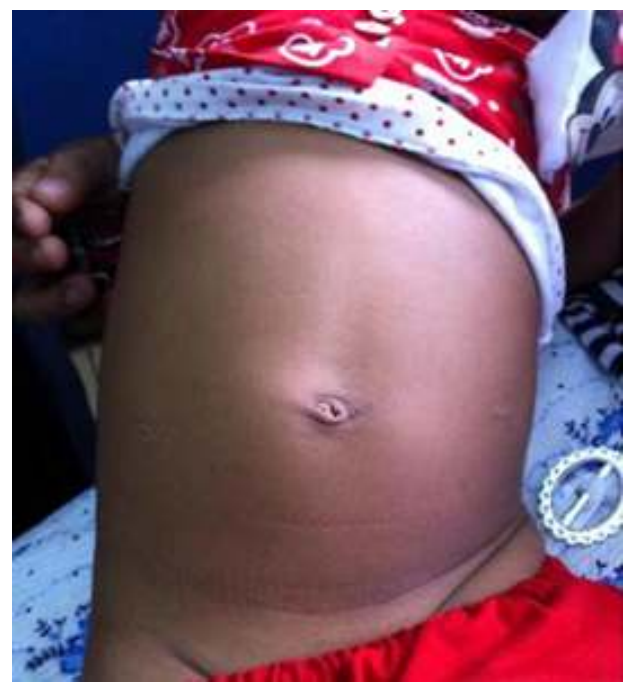

Figure 2: Outcome of laparoscopic primary pullthrough for $H D$ 
In 1948 Swenson $^{1}$ first described the staged surgical procedure for HD. Since then the described technique continued to evolve with slight modifications till the primary laparoscopic pull-through procedure (PLPP) was described by Keith E. Georgeson in $1995^{2,3}$ with modifications introduced in $2008^{4}$. This technique has been widely adopted with good outcome on mid to long term follow up 5 .

Prior to this study PLPP was not practised in Sri Lanka. We describe herein a primary laparoscopic pull-through procedure that was successfully employed to treat twelve infants with HD. This report will highlight our experience and the early results of the above cases. Our experience demonstrates that this minimally invasive surgical procedure is feasible for young infants and we believe that HD may be a definitive indication for laparoscopic surgery.

\section{Method}

The study period extends from April 2010 to May 2011. Twelve patients with histologically diagnosed uncomplicated HD below the age of 4 years were considered candidates for this study. All the surgeries were carried out using the same technique under the supervision of the author. All patients were followed up and closely monitored at the surgical clinic. Parents of these children were called up and interviewed prior to this publication to assess their level of satisfaction with regard to bowel habits and quality of life pre and post operatively.

Prior to surgery the patients were put on a liquid diet and lactulose for one week followed by 10 hours fasting before surgery. In addition, regular bowel washes with normal saline were given for 5 days and the response was assessed by reduction of abdominal distension and $\mathrm{x}$-ray. Polyethylene glycol solution was administered during the day preceding the surgery.

Surgery was performed under the cover of prophylactic antibiotics comprising cefuroxime and metronidazole. General anaesthesia was given with intubation, caudal block and local anaesthesia to port sites. Patient was positioned obliquely/transversely on the table with the head end tilted down to support operating from the cephalic end. Four-port video laparoscopy was performed with a single $10 \mathrm{~mm}$ main port (0 degree telescope) and three $5 \mathrm{~mm}$ accessory ports (Figure 3 ).

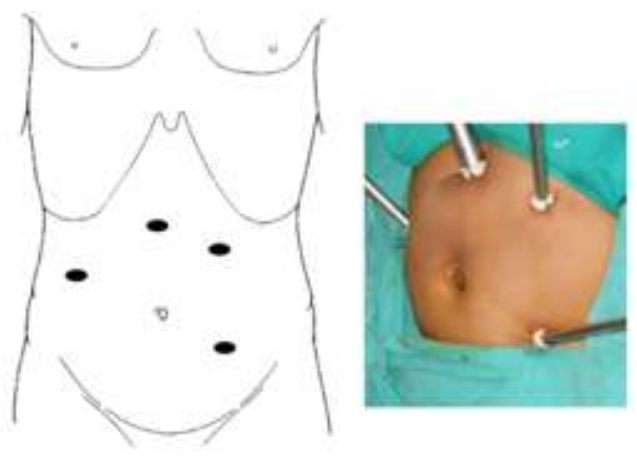

Figure 3: Port sites

After the identification of the transition zone a seromuscular biopsy was obtained with laparoscopic scissors and sent for frozen section histology. The dissection of the aganglionic segment of the distal colon and rectum was done circumferentially up to one to two centimetres below the peritoneal reflection. The perineal dissection commenced following histological confirmation of the transition zone. The rectum and distal colon were pulled down the anus in continuity until the biopsy site was pulled beyond the aganglionic bowel at the point of intended bowel transection. The circumferential transection of the colon was done and one layer anastomosis was fashioned between the neo-rectum and anus using PDS sutures over a rectal tube.

\section{Results}

The mean age at operation was 13 months (range 336 months). Mean duration of surgery was 150 minutes. Mean blood loss was $<10 \mathrm{ml}$. No intraoperative, immediate post-operative or early followup complications requiring re-admission were seen apart from fever lasting $<24$ hours post operatively and frequent stooling. Mean hospital stay was 6 days.

A substantial improvement of bowel habits and general wellbeing was expressed by parents during the call up interview prior to this publication.

\section{Discussion}

HD is a common cause of intestinal obstruction during infancy and the neonatal period. The timehonoured approach was to perform a preliminary colostomy within the neonatal period and subsequent definitive pullthrough at a later date. PLPP can be performed safely during infancy and most of the babies were fed on the $2^{\text {nd }}$ postoperative day and discharged within a week. Therefore PLPP has the advantage of shorter hospital stays, less hospital admissions and less cost to the patient's family and the hospital. Furthermore, it avoids the social, 
psychological and cosmetic morbidity along with extremely satisfactory postoperative faecal continence.

\section{Conclusions}

Early results from our study, as well as the long and midterm results from elsewhere in the world, suggests that PLPP is superior to the traditional staged procedure. Therefore it can be safely concluded that PLPP is a better, safer and effective approach to treat HD. This minimally invasive technique is technically feasible and safe. Thus, the laparoscopic surgery of Hirschsprung disease will progress through a series of technical advances, and may take the place of the standard open operation for this disease in the near future.

\section{References}

1. Swenson O, Bill AH. Resection of rectum and rectosigmoid with preservation of the sphincter for benign spastic lesions producing megacolon. Surgery 1948; 24:212-20.
2. Georgeson KE, Fuenfer MM, Hardin WD. Primary laparoscopic pullthrough for Hirschsprung disease in infants and children. $J$ Pediatr Surg 1995; 30:1-7. http://dx.doi.org/10.1016/0022-3468(95)90333$\underline{X}$

Gerogeson KE, Cohen RD, Hebra A, et al. Primary laparoscopic endorectal colon pullthrough for Hirschsprung disease: a new gold standard. Ann Surg 1999; 229: 678-82. http://dx.doi.org/10.1097/00000658-199905000$\underline{00010}$

3. Gerogeson KE, Muensterer OJ. Laparoscopicassisted transanal pull-through for Hirschsprung Disease, Holschnider AM and Prem Puri (editors), Hirschsprung disease and allied disorders, 3rd ed. Springer: 2008. Ch.23, 323-33

4. Jona JZ. Laparoscopic pull through for Hirschsprung disease in infants. $J$ Indian Assoc Pediatr Surg 2005; 10:28-30.

http://dx.doi.org/10.4103/0971-9261.16072 